\title{
Biphasic response of endothelial progenitor cell proliferation induced by high glucose and its relationship with reactive oxygen species
}

\author{
Wei Zhang ${ }^{1}$, Xin-Hong Wang ${ }^{1}$, Si-Feng Chen ${ }^{1}$, Guo-Ping Zhang ${ }^{2}$, Ning Lu ${ }^{1}$, Ren-Ming Hu ${ }^{3}$ \\ and Hui-Ming Jin ${ }^{1,3}$ \\ ${ }^{1}$ Department of Physiology and Pathophysiology, Shanghai Medical College, ${ }^{2}$ Institutes of Biomedical Sciences, Fudan University, PO Box 224, Shanghai \\ 200032, China \\ ${ }^{3}$ Department of Endocrinology, Institute of Endocrinology and Diabetology at Fudan University, Hua Shan Hospital, Fudan University, Shanghai 200040, China \\ (Correspondence should be addressed to H-M Jin; Email: hmjin@shmu.edu.cn; R-M Hu; Email: renminghu@fudan.edu.cn)
}

\begin{abstract}
In this study, the effect of high glucose (HG) on endothelial progenitor cell (EPC) proliferation and its relationship with cyclins and reactive oxygen species (ROS) were investigated. Mouse EPCs were isolated from bone marrow using a magnetic activated cell-sorting system and cultured in the presence or absence of $\mathrm{HG}(30 \mathrm{mmol} / \mathrm{l})$. We found that in the early stage of incubation ( 3 days), HG promoted cell proliferation, and increased the expressions of cdk2 and cyclin E, while in the late stage of culture (7 days) it inhibited cell proliferation and decreased the expressions of $\mathrm{cdk} 2$, cyclin $\mathrm{E}$, and proliferating cell nuclear antigen (PCNA). Moreover, on the third day after incubation, HG significantly inhibited the apoptosis of EPCs, while in the late stage it markedly
\end{abstract}

activated caspase- 3 and promoted apoptosis. ROS generation in cells and maleic dialdehyde level in medium were significantly increased in HG group on the seventh day, whereas the expressions of superoxide dismutase and glutathione levels decreased. Tempol, a membrane-permeable radical scavenger, significantly inhibited ROS production in EPCs and partially reversed the HG-mediated inhibition of EPCs proliferation on the seventh day. We hypothesize that in the $\mathrm{HG}$ environment, the biphasic response of $\mathrm{EPC}$ proliferation may be related to the generation of ROS, which causes modulation of cyclins and cell cycle effect.

Journal of Endocrinology (2008) 197, 463-470

\section{Introduction}

Endothelial progenitor cells (EPCs) derived from bone marrow can be further differentiated into endothelial cells. EPCs maintain the normal endothelial function of vessels in adults through re-endothelization and angiogenesis. Thus, it plays an important role in the repair process of vascular endothelial cell after its lesion (Urbich \& Dimmeler 2004).

It has already been found (Tepper et al. 2002, Fadini et al. 2006) that in patients with type 2 diabetes mellitus (DM), especially with the complications of vascular disease, the number of EPCs is significantly reduced in peripheral blood, and the functions of EPC are impaired. Therefore, it is proposed that both the mechanism of diabetic vascular disease and the impairment of vascular endothelial repair after its lesion in DM patients might be associated with the EPC functional disorders (Fadini et al. 2005, Zhou et al. 2006).

Since an elevated blood glucose level is the major characteristic of DM, it is important to investigate the change of EPC proliferation under the effect of high glucose (HG) and its relationship with cell cycle, apoptosis, and reactive oxygen species (ROS) production. This may help to further understand the mechanism of diabetic vascular complications and to prevent the vascular complications of DM.

\section{Materials and Methods}

\section{Materials}

Dulbecco's modified Eagle's medium (DMEM) was purchased from Hyclone (Salt Lake, UT, USA). Fetal bovine serum (FBS) and fibronectin (FN) were from Gibco. FicollPaque was from Amersham Biosciences. Vascular endothelial growth factor (VEGF), basic fibroblast growth factor (bFGF), and insulin-like growth factor (IGF) were obtained from Cytolab Ltd (Rehovot, Israel). CD117-PE, CD34-FITC, Flk-1-APC, and Cyclin E antibodies were from eBioscience, Inc. (San Diego, CA, USA). cdk2 and caspase-3 antibodies were from Santa Cruz Biotechnology, Inc. (Santa Cruz, CA, USA). PCNA antibody was from Thermo Fisher Scientific, Inc. (Fremont, CA, USA). 3-(4,5-dimethylthiazol-2-yl)-2,5diphenyl-2H-tetrazolium bromide (MTT), BS-1 lectin, glucose, $2^{\prime}, 7^{\prime}$-dichlorofluorescin diacetate (DCFH-DA), and tempol were from Sigma-Aldrich. 1,1-dioctadecyl-3,3,3,3- 
tetramethylindocarbocyanine(Dil)-labeled acetylated low density lipoprotein (Dil-ac-LDL) was from Molecular Probes, Inc. (Carlsbad, CA, USA). CD117 MicroBead Kit and magnetic activated cell-sorting system (MACS) were from Miltenyi Biotec (Bergisch Gladbach, Germany). Annexin$\mathrm{V} / \mathrm{PI}$ kit was from Bender MedSystems Inc. (Burlingame, CA, USA). Maleic dialdehyde (MDA) and glutathione (GSH) kits were from Nanjing Jiancheng Bioengineering Institute (Nanjing, China).

\section{CD117+ cells isolation and induction}

$\mathrm{CD} 117+$ cells were isolated from BALB/C mice (2-3 month) as described previously (Zhang et al. 2006). The animal protocol was approved by animal committee of Fudan University. Mouse bone marrow cells were isolated from the femora and tibiae. Mononuclear cells were separated by density centrifugation through Ficoll-Paque at $400 \mathrm{~g}$ and for $25 \mathrm{~min}$. After washing, the cells were resuspended and incubated with $20 \mu \mathrm{l} / 10^{7}$ cells of MicroBead containing anti-mouse CD117 monoclonal antibody at $4{ }^{\circ} \mathrm{C}$ for $30 \mathrm{~min}$. CD117 + cells were isolated by MACS.

CD117+ cells $\left(2 \times 10^{5} / \mathrm{cm}^{2}\right)$ were plated in a 24-well plate coated with FN. One milliliter of DMEM containing $20 \%$ FBS was added into each well combined with $20 \mathrm{ng} / \mathrm{ml}$ VEGF, $5 \mathrm{ng} / \mathrm{ml} \mathrm{bFGF}$, and $20 \mathrm{ng} / \mathrm{ml}$ IGF. The cells were maintained in a humidified $5 \% \mathrm{CO}_{2}$ incubator at $37^{\circ} \mathrm{C}$. The fresh medium was changed every $3-4$ days until 7 or 9 days.

\section{Phagocytotic activity assay}

Direct fluoroscent staining was used to detect dual binding of fluoroscein isothiocyanate (FITC)-labeled BS-1 lectin and Dil-ac-LDL. Cells demonstrating double-positive fluorescence were identified as differentiating EPCs. The detailed method was described in our previous report (Zhang et al. 2006).

\section{Flow cytometry analyses}

The proportion of cells positive for CD117, CD34, and Flk-1 was determined by flow cytometry according to our routine method (Zhang et al. 2006).

\section{MTTassay}

Cell proliferation and viability were determined by an MTT assay as reported previously (Zhang et al. 2006).

\section{Cell cycle analysis}

EPCs were harvested by trypsinization, washed twice with PBS, and fixed with ethanol. After RNAse treatment and staining with propidium iodide (PI), DNA content was measured by flow cytometry (Becton Dickinson, San Diego, CA, USA).

\section{Proliferation index}

Based on the results of cell cycles analysis, the proliferation index is expressed as the result of the following equation (Wang \& Ren 2006).

$\mathrm{PI}=\frac{\mathrm{S}+\mathrm{G} 2 / \mathrm{M}}{\mathrm{G} 0 / \mathrm{G} 1+\mathrm{S}+\mathrm{G} 2 / \mathrm{M}} \times 100 \%$

\section{ROS generation}

Intracellular ROS generation was assessed using DCFH-DA and analyzed by flow cytometry as described previously (Ho et al. 2000). DCFH-DA is converted by intracellular esterase to DCFH, which is oxidized into the highly fluorescent dichlorofluorescin (DCF) in the presence of a proper oxidant, and then analyzed by flow cytometry.

\section{Determination of lipid peroxides and scavengers}

The MDA content and GSH level in the medium were measured using their respective kits. Superoxide dismutase (SOD) content was measured by western blot.

\section{Measurement of apoptosis}

Effect of HG on apoptosis of EPC was evaluated using the Annexin-V/PI kit. EPCs were washed twice in PBS and resuspended in binding buffer $\left(2-5 \times 10^{5}\right.$ cells $\left./ \mathrm{ml}\right)$. About $195 \mu \mathrm{l}$ cells were incubated with $5 \mu \mathrm{l}$ Annexin-V/FITC for $10 \mathrm{~min}$ at room temperature, washed, incubated with $10 \mu \mathrm{l}$ PI solution in $200 \mu \mathrm{l}$ binding buffer and afterwards analyzed by flow cytometry. Caspase- 3 was detected by western blot using the anti-caspase- 3 antibody specific for the $32 \mathrm{kDa}$ cleaved fragment.

\section{Determination of cellular cyclins}

Cdk2, cyclin E, and PCNA were determined by western blot analysis. The total protein of EPCs was extracted and its concentration was measured using BCA methods. The extracted protein was subjected to SDS-PAGE gel for electrophoresis and then transferred to PVDF membrane. The membrane was blocked for $2 \mathrm{~h}$ with TBST (tris-buffered saline with $0 \cdot 05 \%$ tween-20) blocking solution containing 5\% skimmed milk in TBST, and incubated overnight at $4{ }^{\circ} \mathrm{C}$ with anti-cdk2 polyclonal antibody, anti-cyclin $\mathrm{E}$ polyclonal antibody, anti-PCNA monoclonal antibody, and then horseradish peroxidase (HRP)-conjugated goat anti-rabbit or rabbit anti-mouse immunoglobulin G antibody (1: 5000 
dilution) for $30 \mathrm{~min}$ at $37^{\circ} \mathrm{C}$ followed by enhanced chemiluminescence (ECL) visualization of the bands.

\section{Statistical analysis}

A statistical package SPSS 10.0 (SPSS incorporated, Chicago, IL, USA) was used for all analyses. All experiments were performed at least in triplicate. All values were expressed as mean \pm s.D., and the ANOVA was used to determine the significance of differences among the groups. Values with $P<0.05$ were considered statistically significant.

\section{Results}

\section{Characteristics of EPCs}

Under light microscope, cells changed from globe-like shape (at inoculation) to thin and flat, then round, fusiform, pebblelike shape (7-10 days), string - even luminal structure on the 14th day when cells were arranged in a linear manner.

Flow cytometric measurement showed that the percentage of CD117+ CD34 + Flk-1 + cells was $52 \cdot 3 \pm 4 \cdot 7 \%$ after CD117 magnetic cell sorting.
Seven days after culture of CD117+ cells, DiIac-LDL/BS-1-Lectin double-positive cells were $93 \cdot 2 \pm 8 \cdot 6 \%$.

\section{Effect of HG on EPC proliferative activity}

MTT measurements were taken on the first, third, fifth, seventh, and ninth day of the culture. Results showed that the cell proliferative activity of $\mathrm{HG}$ group $(30 \mathrm{mmol} / 1$ glucose; Seeger et al. 2005, Sheu et al. 2005) was significantly higher than that of the control group $(5 \mathrm{mmol} / 1$ glucose plus $25 \mathrm{mmol} / \mathrm{l}$ mannitol) on the third day while it was significantly lower than that of the control group on the seventh and ninth day (Fig. 1A).

\section{Cell counting}

The results showed that the number of double-positive fluorescence cells (i.e. differentiating EPCs) in HG group was significantly higher than that of the control group on the third day, while it was markedly lower than that of the control group on the seventh and ninth day. There was no significant difference between the two groups at other time points (Fig. 1B).
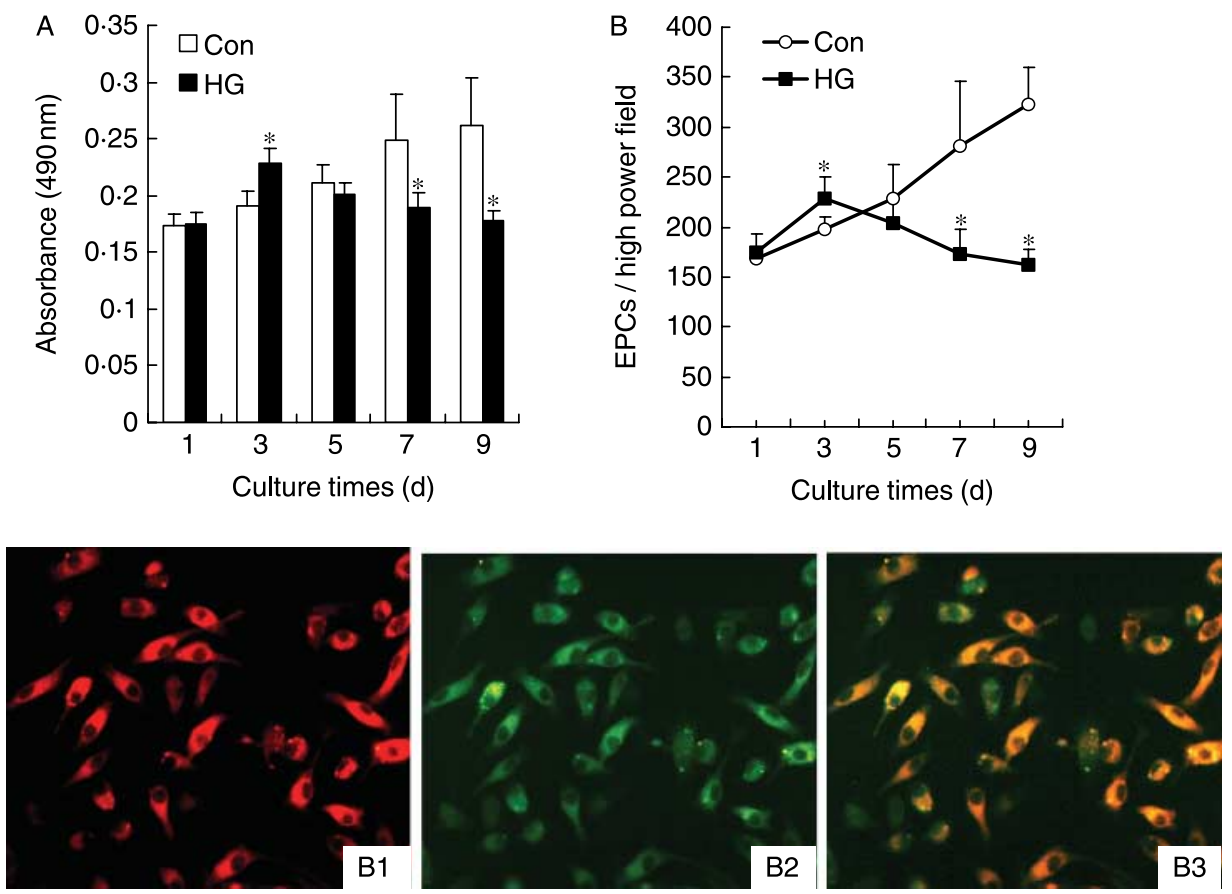

Figure 1 Effects of high glucose on EPC proliferation. (A) MTT assays showed that the EPC proliferative activity in the high glucose group increased on the third day, while significantly reduced on the seventh and ninth day compared with the control group $\left({ }^{*} P<0 \cdot 05\right)$. (B) Phagocytotic activity assay showed that the positive EPC number significantly increased in the high glucose group compared with the control group on the third day, while markedly decreased on the seventh and ninth day $\left({ }^{*} P<0 \cdot 05\right)$. Dil-ac-LDL and BS-1Lectin double-positive cells were EPCs undergoing differentiation (B1, Dil-ac-LDL positive cells; B2, BS-1Lectin positive cells; B3, double-positive cells). 
Effect of HG on EPC proliferation index

The cell proliferation index of the HG group was significantly higher than that of the control group on the third day $(0 \cdot 3307 \pm 0 \cdot 0683$ vs $0 \cdot 2607 \pm 0 \cdot 0285, P<0 \cdot 05)$, while was markedly lower than that of the control group on the seven day $(0 \cdot 2064 \pm 0 \cdot 0220$ vs $0 \cdot 2699 \pm 0 \cdot 0420, P<0 \cdot 05)$.

\section{Effect of HG on cell cycle distribution}

The cell cycle analysis showed that the percentage of G2/M stage cells of HG group was significantly higher than that of the control group on the third day. By contrast, on the seventh day, the cells were markedly arrested at the G0/G1 phase and $\mathrm{S}$ stage cells were significantly decreased in HG group compared with the control group (Table 1).

\section{Measurement of EPC apoptosis and caspase-3 protein}

The percentage of apoptosis in the HG group was lower than that of the control group on the third day (Fig. 2A). Interestingly, only early stage apoptosis (Annexin,$+ \mathrm{PI}-$ ) appeared to be decreased whereas no significant changes in late stage apoptosis (Annexin,$+ \mathrm{PI}+$ ) was detected. On the other hand, the percentage of apoptosis in the HG group was significantly increased compared with the control group on the seventh day (Fig. 2B). Moreover, the increase was only detected for early stage apoptosis.

Western blot analysis indicated that the cleaved caspase- 3 in HG group was not significantly changed when compared with that in the control group on the third day, while it was significantly increased when compared with that in the control group on the seventh day (Fig. 2C).

Effects of HG on expressions of cyclins

The expressions of cdk2 and cyclin E in HG group were significantly higher than that of the control group on the third day. On the other hand, there was no significant difference in the expression of PCNA between the two groups on the third day. However, the expressions of $\mathrm{cdk} 2$, cyclin E, and PCNA in $\mathrm{HG}$ were markedly lower than that of the control group on the seventh day (Fig. 3).

Table 1 The effect of high glucose on cell cycle of endothelial progenitor cells (EPCs)

\begin{tabular}{|c|c|c|c|c|}
\hline & Group & G0-G1 stage & G2-M stage & S stage \\
\hline \multicolumn{5}{|l|}{ Days } \\
\hline \multirow[t]{2}{*}{3} & Con & $73 \cdot 35 \pm 8 \cdot 52$ & $1 \cdot 21 \pm 0 \cdot 54$ & $25 \cdot 43 \pm 8 \cdot 68$ \\
\hline & $\mathrm{HG}$ & $67 \cdot 61 \pm 7 \cdot 35$ & $3 \cdot 49 \pm 1 \cdot 05^{*}$ & $28.67 \pm 4.42$ \\
\hline \multirow[t]{2}{*}{7} & Con & $71 \cdot 69 \pm 4 \cdot 50$ & $1 \cdot 44 \pm 1 \cdot 08$ & $26 \cdot 32 \pm 4 \cdot 64$ \\
\hline & $\mathrm{HG}$ & $80 \cdot 58 \pm 3.94 *$ & $1 \cdot 39 \pm 1 \cdot 25$ & $18 \cdot 81 \pm 3 \cdot 28^{*}$ \\
\hline
\end{tabular}

${ }^{*} P<0 \cdot 05$ versus Con. Con, control group; $\mathrm{HG}$, high glucose group.
Effect of HG on ROS generation in EPCs

There was no significant difference in the DCF fluorescence intensity (the quantity of ROS generation) in the EPCs between the $\mathrm{HG}$ and the control groups on the third day (Fig. 4A), whereas the DCF fluorescence intensity in the EPCs of the HG group was significantly increased when compared with the control group on the seventh day (Fig. 4B).

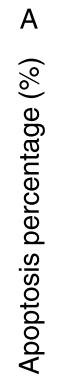
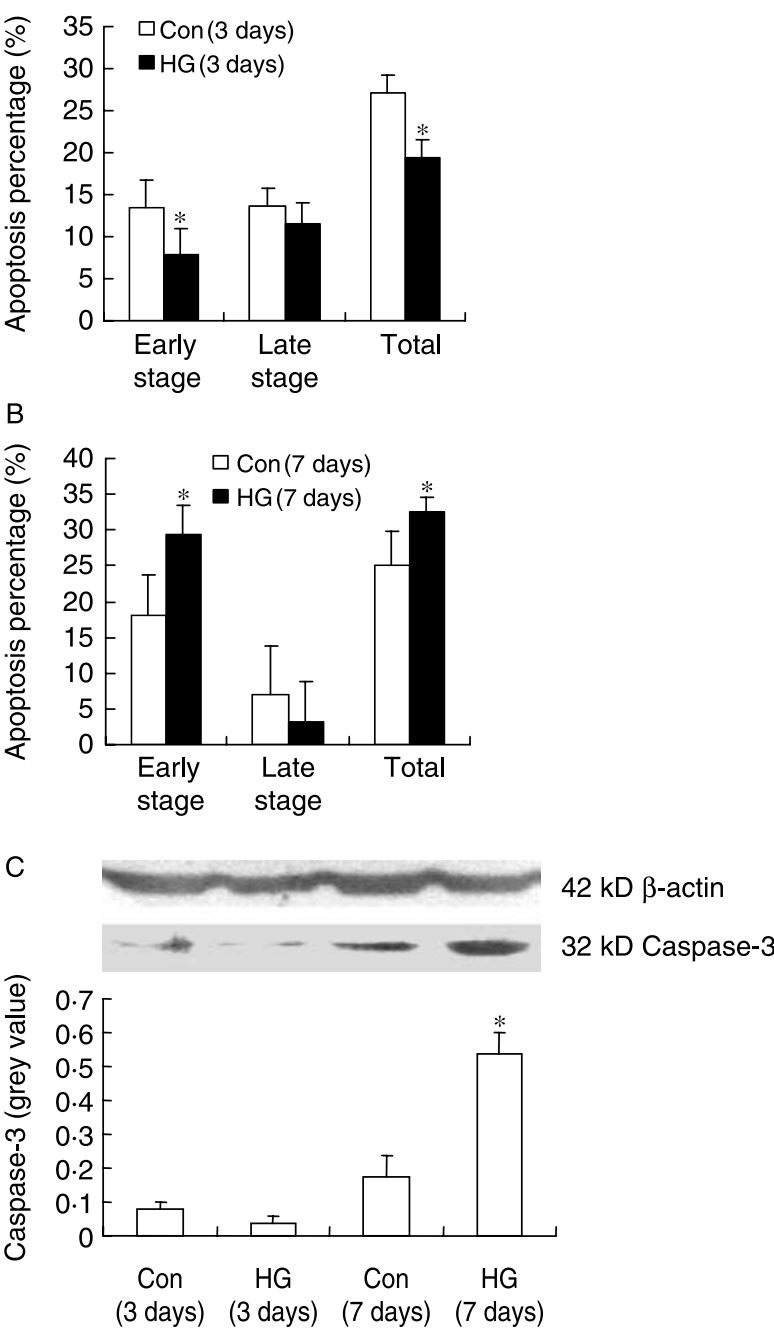

Figure 2 Effects of high glucose on EPC apoptosis. The percentage of apoptosis was determined by flow cytometry after FITC-labeled Annexin V/PI double staining. (A) The percentage of apoptosis in the high glucose group significantly decreased compared with the control group on the third day, and the decrease of apoptosis was predominantly at early stage $\left({ }^{*} P<0 \cdot 05\right)$. (B) There was an increase of apoptosis compared with the control group, particularly early stage apoptosis on the seventh day $\left({ }^{*} P<0 \cdot 05\right)$. (C) Western blots indicated no significant difference in cleaved caspase-3 between high glucose and control groups on the third day, but a significant increase in high glucose group on the seventh day $\left({ }^{*} P<0 \cdot 05\right)$. 


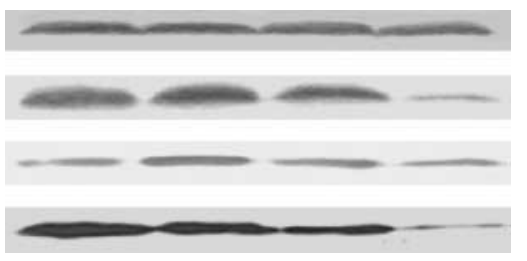

\section{$42 \mathrm{kD} \quad \beta$-actin \\ $35 \mathrm{kD} \quad \mathrm{Cdk} 2$ \\ $50 \mathrm{kD}$ Cyclin E \\ 36 kD PCNA}

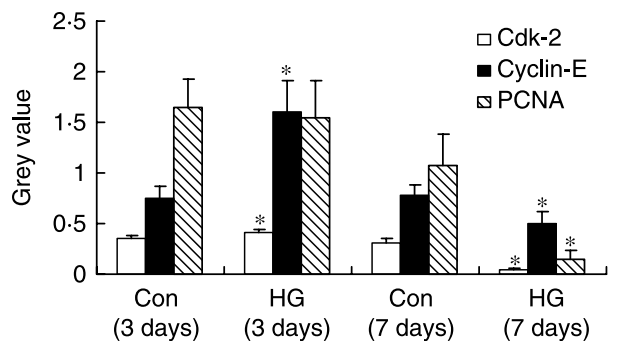

Figure 3 Effects of high glucose on expressions of cyclins.

The expressions of cdk2 and cyclin E in high glucose (HG) group were significantly increased compared with the control group on the third day $\left({ }^{*} P<0 \cdot 05\right)$. There was no significant difference in the expression of PCNA between the two groups on the third day $(P>0 \cdot 05)$. However, the expressions of cdk2, cyclin E, and PCNA were markedly decreased in HG compared with the control group on the seventh day $\left({ }^{*} P<0 \cdot 05\right)$.

\section{Changes of SOD, GSH, and MDA}

The expression of SOD in HG group was not significantly changed on the third day compared with that of the control group, but was significantly decreased compared with the control group on the seventh day (Fig. 4C).

The GSH content was decreased and MDA content was significantly increased in medium in HG group compared with the control group on the seventh day (Table 2).

\section{Effects of tempol on the ROS production and EPCs proliferation}

Next, we evaluated the effect of tempol on ROS generation in EPCs. A tempol concentration of $1 \mathrm{mmol} / 1$ was selected based on previous reports (Chatterjee et al. 2000). EPCs were pretreated with tempol at $37^{\circ} \mathrm{C}$, and $20 \mathrm{~min}$ later, they were exposed to $\mathrm{HG}$ environment for 7 days. Extra tempol treatment on EPCs was performed while the medium was changed at third day. Then FACS was used to detect the change of DCF fluorescence intensities (which are also the quantity of ROS generation) in the EPCs of different groups. Results showed that the ROS generation in the EPCs of $\mathrm{HG}$ plus tempol group was significantly lower than that of HG group (Fig. 5A). On the other hand, the proliferative activity and proliferation index of EPCs in HG plus tempol group were significantly higher than that of the HG group (Fig. 5B and C). The cell cycle analysis showed that the percentage of $\mathrm{S}$ stage cells in HG plus tempol group was significantly higher than that of the HG, while the percentages of G0/G1 stage and G2/M stage cells were not of significant difference between the two groups
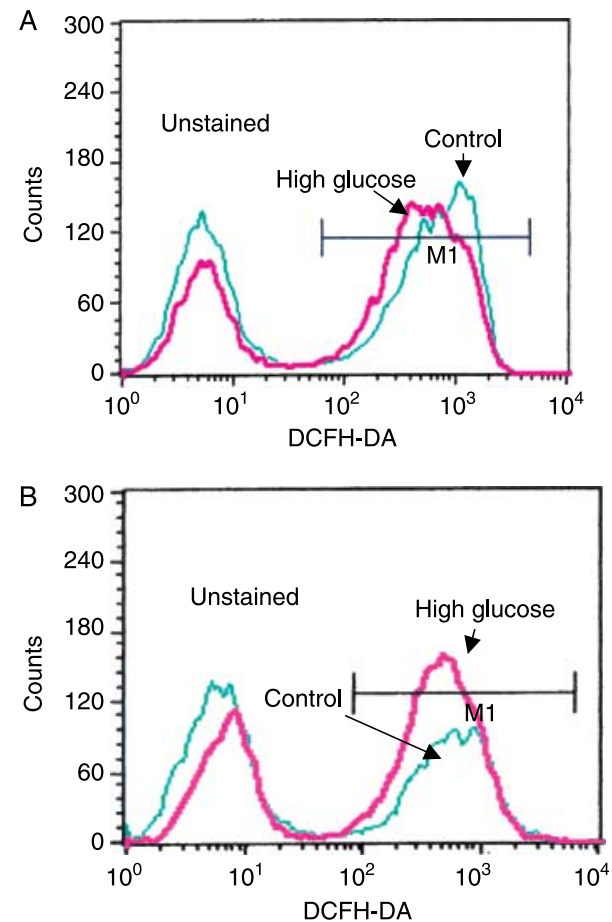

C
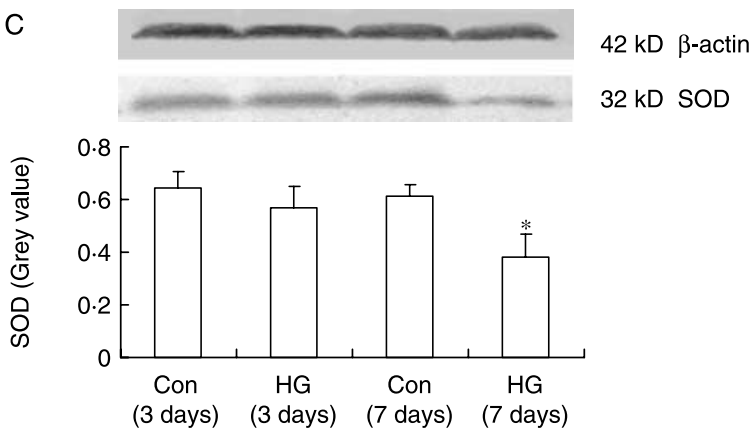

Figure 4 Effects of high glucose on ROS generation in EPCs. Intracellular ROS generation was determined using DCFH-DA and analyzed by flow cytometry. (A) There was no significant difference in the quantity of ROS generation in the EPCs between the high glucose and control groups on the third day $(P>0 \cdot 05)$. (B) The quantity of ROS generation in the EPCs in high glucose group significantly increased compared with the control group on the seventh day $(P<0 \cdot 05)$. (C) The expression of SOD in high glucose group did not show an obvious change on the third day compared with the control group, while it was significantly decreased on the seventh day $\left({ }^{*} P<0 \cdot 05\right)$.

(Table 3). These data suggest that the tempol, by lowering ROS production, could partly meliorate the inhibition of HG on EPCs proliferation on the seventh day.

\section{Discussion}

It is reported that circulating EPCs are reduced in peripheral vascular complications of type 2 DM (Fadini et al. 2005). 
Table 2 The effect of high glucose on maleic dialdehyde (MDA) and glutathione $(\mathrm{GSH})$ in medium

\begin{tabular}{|c|c|c|c|}
\hline & Group & MDA $(\mathrm{nmol} / \mathrm{ml})$ & $\mathbf{G S H}(\mathrm{mg} / \mathrm{ml})$ \\
\hline \multicolumn{4}{|c|}{ Days } \\
\hline \multirow[t]{2}{*}{3} & Con & $2 \cdot 10 \pm 0 \cdot 37$ & $0 \cdot 19 \pm 0 \cdot 05$ \\
\hline & HG & $1 \cdot 93 \pm 0 \cdot 42$ & $0 \cdot 21 \pm 0 \cdot 04$ \\
\hline \multirow[t]{2}{*}{7} & Con & $2 \cdot 40 \pm 0 \cdot 21$ & $0 \cdot 20 \pm 0 \cdot 03$ \\
\hline & HG & $4 \cdot 60 \pm 0.53^{*}$ & $0 \cdot 15 \pm 0 \cdot 01^{*}$ \\
\hline
\end{tabular}

${ }^{*} P<0.05$ versus Con. Con, control group; HG, high glucose group.

Tamarat $e t$ al. demonstrated a reduced angiogenic potential of EPCs in diabetic animals. Administration of diabetic progenitor cells into the ischemic hindlimbs of non-diabetic mice improved the angiogenic response to a lesser extent (Tamarat et al. 2004). Moreover, Tepper et al. (2002) reported that the proliferation and capillary tube formation of EPCs were impaired in patients with type 2 diabetes compared with normal subjects. Although these studies have suggested an adverse effect of DM on the functional activity of EPCs, the underlying mechanisms remain unsolved.

Since an elevated level of blood glucose is the major characteristic of DM, it is important to explore the change of EPC function under the effect of HG. Marchetti et al. demonstrated that HG significantly affected the number of EPC colony forming units, the uptake and binding of ac-LDL and lectin-1, and the ability to differentiate into $\mathrm{CD} 31+$ and vascular endothelial growth factor receptor 2-positive cells. Functional analysis indicated a reduced EPC involvement in de novo capillary tube formation, when co-cultured with mature endothelial cells (human umbilical vein endothelial cells) on matrigel (Marchetti et al. 2006). In addition, hyperglycemia reduces the survival and impairs the migrational and integrative capacities of circulating blood-derived progenitor cells (Krankel et al. 2005).

However, there were very fewer studies on the effect of $\mathrm{HG}$ on the proliferation of EPCs as well as the underlying mechanisms. Recently, it was reported that the proliferation of EPCs in peripheral blood was significantly inhibited by HG at seventh-tenth day, suggesting that HG may accelerate EPC senescence (Kuki et al. 2006). In the current study, we investigated the effect of a single factor HG on the proliferation of EPCs in vitro and explored the

Table 3 The effect of tempol on cell cycle at seventh day of culture

\begin{tabular}{|c|c|c|c|}
\hline \multirow[b]{2}{*}{ Group } & G0-G1 stage & G2-M stage & S stage \\
\hline & & & \\
\hline Con & $71 \cdot 48 \pm 3 \cdot 70$ & $1 \cdot 49 \pm 1 \cdot 08$ & $26 \cdot 82 \pm 4 \cdot 30$ \\
\hline $\mathrm{HG}$ & $80 \cdot 83 \pm 2 \cdot 70^{*}$ & $1 \cdot 37 \pm 1 \cdot 02$ & $18.06 \pm 2.00 *$ \\
\hline $\mathrm{HG}+\mathrm{T}$ & $75 \cdot 89 \pm 3 \cdot 79$ & $1.39 \pm 0.63$ & $21 \cdot 91 \pm 1 \cdot 74^{+}$ \\
\hline
\end{tabular}

${ }^{*} P<0 \cdot 05$ versus Con. ${ }^{\dagger} P<0 \cdot 05$ versus HG. Con, control group; HG, high glucose group; $\mathrm{HG}+\mathrm{T}$, high glucose plus tempol.
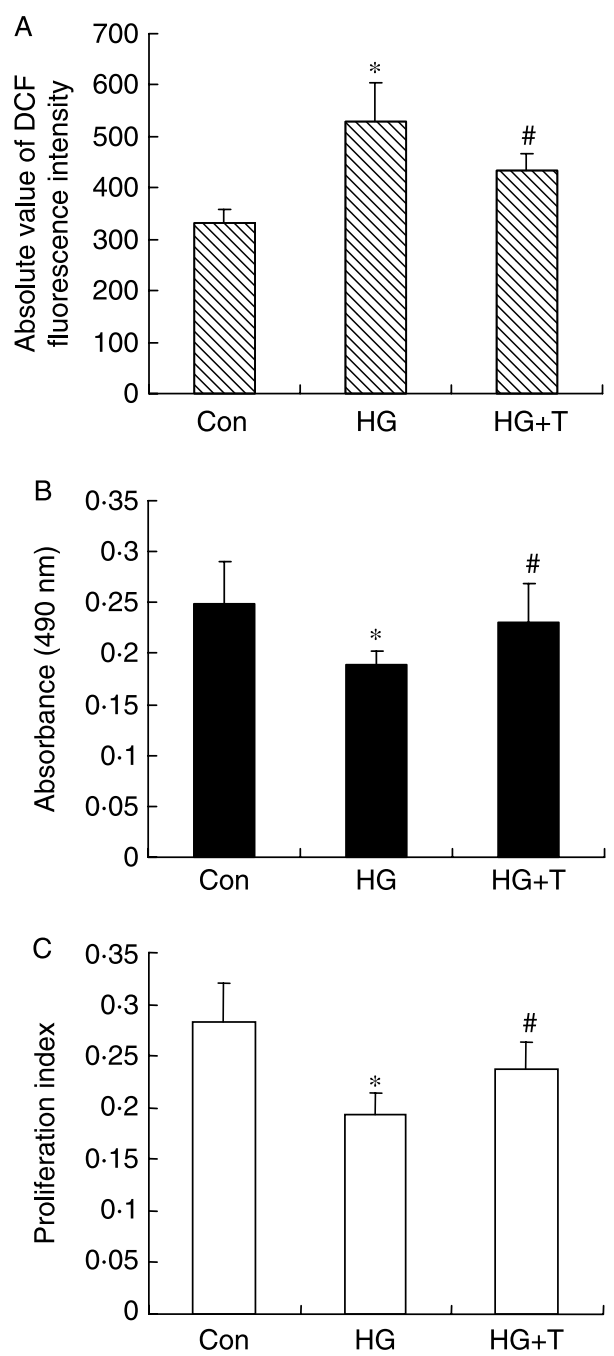

Figure 5 Effect of tempol on the ROS production and EPCs proliferation. (A) The quantity of ROS generation in the EPCs in the high glucose plus tempol $(\mathrm{HG}+\mathrm{T})$ group significantly decreased compared with the high glucose group on the seventh day $\left({ }^{P} P<0 \cdot 05\right)$. (B and $\mathrm{C}$ ) The EPC proliferative activity and proliferative index in the high glucose plus tempol $(\mathrm{HG}+$ $\mathrm{T})$ group markedly increased compared with the high glucose group on the seventh day $\left({ }^{\sharp} P<0 \cdot 05\right)$.

possible mechanisms. In this report, we used $5 \mathrm{mmol} / \mathrm{l}$ D-glucose as a control because this is the normal concentration in DMEM and is equivalent to the serum glucose concentration in normal individuals $(90 \mathrm{mg} / \mathrm{dl})$. D-glucose $(30 \mathrm{mmol} / \mathrm{l})$ was used as the challenge dose because this reflects a high serum glucose concentration in DM individuals $(540 \mathrm{mg} / \mathrm{dl})$. Mannitol was chosen as an osmolar control because it is known to have no direct impact on glucose metabolism. EPCs were exposed to prolonged HG environment (1-9 days). We observed the change of EPC proliferation under HG. The MTT assay revealed a biphasic effect of $\mathrm{HG}$ on proliferative activity of 
EPCs. It promoted cell proliferation for the initial 3 days, but inhibited EPC growth thereafter (on the seventh and ninth day). Consistently, on the third day, the EPC count and cell proliferation index in HG group were higher than that of the control group, whereas on the seventh day, cell count and proliferation index were markedly lower than that of the control group. Similar observations were made previously on other cell types such as human umbilical vein endothelial cells. Under certain pathological conditions, HG treatment induced a biphasic response in cell growth, i.e. an early cell proliferation and a late cell apoptosis (Pascal et al. 1996, Ho et al. 2006).

In order to understand the mechanism of biphasic response of EPC proliferation to $\mathrm{HG}$, we investigated the effect of HG on cell cycle distribution. Our findings showed that the percentage of G2/M stage EPCs in the HG group was significantly increased when compared with that in the control group on the third day, whereas on the seventh day the percentage of G0/G1 stage EPCs in the HG group was markedly increased and S-stage EPCs was significantly decreased. In addition, we found that expression levels of cdk2 and cyclin E in HG group were significantly increased on the third day, but on the seventh day, the expression levels of cdk2, cyclin E as well as PCNA were all reduced. Cyclin E is known to contribute to proliferation in normal cells by accelerating the G1-S phase turnover of the cell cycle. Cdk2 is activated by cyclin A and cyclin E, and controls the G1-S phase of the cell cycle by phosphorylation of $\mathrm{pRb}$. The increased expressions of cyclin $\mathrm{E}$ and $\mathrm{cdk} 2$ in $\mathrm{HG}$ group on the third day might explain that HG can accelerate the G1-S phase turnover of the cell cycle and promote the cells proliferation. The reduction of expressions of cyclin E, cdk2, and PCNA in HG group on the seventh day suggests that HG can suppress cell proliferation through blocking G1-S phase transition in cell cycle.

Furthermore, HG also caused biphasic effects on EPC apoptosis, i.e. HG inhibited EPC apoptosis on the third day, but promoted apoptosis on the seventh day. Caspases are the key enzymes that cause cell apoptosis. Once the signal pathway of caspase- 3 is activated, proteins inside cells can be degraded and cell apoptosis irreversibly occurs. Caspase- 3 , as an important member of the caspase family, is the executor of cell apoptosis. In this study the level of cleaved caspase- 3 in EPCs showed no significant change in the HG group in the early stage of incubation (3 days) but was increased significantly in the late stage ( 7 days). These data suggest that inhibition of EPC proliferation by prolonged (7 days) treatment with HG may be partially due to the induction of apoptosis via activation of caspase-3. Only mild change of early stage apoptosis of EPCs was measured in the HG environment and the effect of HG on apoptosis needs to be further clarified in future studies.

HG can induce oxidative stress according to several published studies (Zurova-Nedelcevova et al. 2006, Ksiazek et al. 2007). The data presented in this report suggest that the ROS generation in EPCs in HG group was significantly higher than that in the control group on the seventh day while there was no significant change on the third day. This finding is consistent with the following observations: 1) reduced expression of SOD, an important intracellular antioxidant enzyme detoxifying superoxide anion, 2) higher level of MDA, a lipid peroxidation product that can be induced by ROS on cell membrane, and 3) decreased content of GSH, a major non-protein thiol in cells functioning as a free radical scavenger. On the other hand, we found that addition of exogenous tempol, a membrane-permeable radical scavenger, could significantly inhibit ROS production in the EPCs on the seventh day and partially reversed the HG-mediated inhibition of EPCs proliferation. Taken together, the data presented herein support our hypothesis that the decreased ability of EPC to proliferate following chronic exposure to HG could be caused by an excessive production of ROS in the EPCs. Enhanced ROS production could in turn cause cell arrest by inhibiting expression of certain cyclins.

In summary, we propose that in the chronic course of diabetes, high concentration of glucose inhibits EPC proliferation and such an effect is associated with excessive ROS production. The reduced ability of EPCs as well as other EPC dysfunctions to proliferate could result in compromised ability of endothelial repair and angiogenesis. This may play a central role in the development of diabetic macro-/microangiopathy, decreased collateral arteriogenesis around occlusive lesions, and decreased re-endothelialization of injured blood vessels. Our data suggest that one of the potential causes of diabetic vascular complications is EPC dysfunction induced by ROS during hyperglycemia. Hence, further studies to identify ways to alleviate the HG-induced impairment of EPC functions will be important for the prevention and treatment of diabetic vascular complications.

\section{Acknowledgements}

This work was funded by Research Project of Shanghai Municipal Bureau of Health (No. 054084), Grant from Shanghai Science and Technology Commission (04dz19504), the Key Project of National Natural Science Foundation of China (30230380), and the National Key Basic Research and Development Program (2002CB713703).

\section{Disclosure}

The authors declare that there is no conflict of interest that would prejudice its impartiality.

\section{References}

Chatterjee PK, Cuzzocrea S, Brown PA, Zacharowski K, Stewart KN, MotaFilipe H \& Thiemermann C 2000 Tempol, a membrane-permeable radical scavenger, reduces oxidant stress-mediated renal dysfunction and injury in the rat. Kidney International $\mathbf{5 8}$ 658-673. 
Fadini GP, Miorin M, Facco M, Bonamico S, Baesso I, Grego F, Menegolo M, de Kreutzenberg SV, Tiengo A, Agostini C et al. 2005 Circulating endothelial progenitor cells are reduced in peripheral vascular complications of type 2 diabetes mellitus. Journal of the American College of Cardiology 45 1449-1457.

Fadini GP, Sartore S, Albiero M, Baesso I, Murphy E, Menegolo M, Grego F, Vigili de Kreutzenberg S, Tiengo A, Agostini C et al. 2006 Number and function of endothelial progenitor cells as a marker of severity for diabetic vasculopathy. Arteriosclerosis, Thrombosis, and Vascular Biology 26 2140-2146.

Ho FM, Liu SH, Liau CS, Huang PJ \& Lin-Shiau SY 2000 High glucoseinduced apoptosis in human endothelial cells is mediated by sequential activations of c-Jun $\mathrm{NH}(2)$-terminal kinase and caspase-3. Circulation 101 2618-2624.

Ho FM, Lin WW, Chen BC, Chao CM, Yang CR, Lin LY, Lai CC, Liu SH \& Liau CS 2006 High glucose-induced apoptosis in human vascular endothelial cells is mediated through NF-kappaB and c-Jun NH2-terminal kinase pathway and prevented by PI3K/Akt/eNOS pathway. Cell Signalling 18 391-399.

Krankel N, Adams V, Linke A, Gielen S, Erbs S, Lenk K, Schuler G \& Hambrecht R 2005 Hyperglycemia reduces survival and impairs function of circulating blood-derived progenitor cells. Arteriosclerosis, Thrombosis, and Vascular Biology 25 698-703.

Ksiazek K, Breborowicz A, Jorres A \& Witowski J 2007 Oxidative stress contributes to accelerated development of the senescent phenotype in human peritoneal mesothelial cells exposed to high glucose. Free Radical Biology and Medicine 42 636-641.

Kuki S, Imanishi T, Kobayashi K, Matsuo Y, Obana M \& Akasaka T 2006 Hyperglycemia accelerated endothelial progenitor cell senescence via the activation of $\mathrm{p} 38$ mitogen-activated protein kinase. Circulation Journal $\mathbf{7 0}$ 1076-1081.

Marchetti V, Menghini R, Rizza S, Vivanti A, Feccia T, Lauro D, Fukamizu A, Lauro R \& Federici M 2006 Benfotiamine counteracts glucose toxicity effects on endothelial progenitor cell differentiation via Akt/FoxO signaling. Diabetes 55 2231-2237.

Pascal MM, Knott RM \& Forrester JV 1996 Glucose mediated regulation of transforming growth factor beta in human retinal endothelial cells. Biochemical Society Transactions 24228 S.

Seeger FH, Haendeler J, Walter DH, Rochwalsky U, Reinhold J, Urbich C, Rossig L, Corbaz A, Chvatchko Y, Zeiher AM et al. 2005 p38 mitogenactivated protein kinase downregulates endothelial progenitor cells. Circulation 111 1184-1191.
Sheu ML, Ho FM, Yang RS, Chao KF, Lin WW, Lin-Shiau SY \& Liu SH 2005 High glucose induces human endothelial cell apoptosis through a phosphoinositide 3-kinase-regulated cyclooxygenase-2 pathway. Arteriosclerosis, Thrombosis, and Vascular Biology 25 539-545.

Tamarat R, Silvestre JS, Le Ricousse-Roussanne S, Barateau V, LecomteRaclet L \& Clergue M 2004 Impairment in ischemia-induced neovascularization in diabetes: bone marrow mononuclear cell dysfunction and therapeutic potential of placenta growth factor treatment. American Journal of Pathology 164 457-466.

Tepper OM, Galiano RD, Capla JM, Kalka C, Gagne PJ, Jacobowitz GR, Levine JP \& Gurtner GC 2002 Human endothelial progenitor cells from type II diabetics exhibit impaired proliferation, adhesion, and incorporation into vascular structures. Circulation 106 2781-2786.

Urbich C \& Dimmeler S 2004 Endothelial progenitor cells characterization and role in vascular biology. Circulation Research 95 343-353.

Wang MX \& Ren LM 2006 Growth inhibitory effect and apoptosis induced by extracellular ATP and adenosine on human gastric carcinoma cells: involvement of intracellular uptake of adenosine. Acta Pharmacologica Sinica 27 1085-1092.

Zhang W, Zhang GP, Jin HM \& Hu RM 2006 Characteristics of bone marrow-derived endothelial progenitor cells in aged mice. Biochemical and Biophysical Research Communications 348 1018-1023.

Zhou B, Wu KH, Poon MC \& Han ZC 2006 Endothelial progenitor cells transfected with PDGF: cellular and molecular targets for prevention of diabetic microangiopathy. Medical Hypotheses 67 1308-1312.

Zurova-Nedelcevova J, Navarova J, Drabikova K, Jancinova V, Petrikova M, Bernatova I, Kristova V, Snirc V, Nosalova V \& Sotnikova R 2006 Participation of reactive oxygen species in diabetes-induced endothelial dysfunction. Neuro Endocrinology Letters 27 168-171.

\section{Received in final form 21 February 2008 \\ Accepted 31 March 2008 \\ Made available online as an Accepted Preprint 31 March 2008}

